\title{
Editorial: Recent Advances in Flowering Time Control
}

\author{
Christian Jung ${ }^{1 *}$, Klaus Pillen $^{2}$, Dorothee Staiger ${ }^{3}$, George Coupland ${ }^{4}$ and \\ Maria von Korff ${ }^{4,5}$ \\ ${ }^{1}$ Plant Breeding Institute, Christian-Albrechts-University of Kiel, Kiel, Germany, ${ }^{2}$ Plant Breeding Institute, Martin Luther \\ University of Halle-Wittenberg, Halle, Germany, ${ }^{3}$ Molecular Cell Physiology, Faculty of Biology, Bielefeld University, Bielefeld, \\ Germany, ${ }^{4}$ Department of Plant Developmental Biology, Max Planck Institute for Plant Breeding Research, Cologne, \\ Germany, ${ }^{5}$ Cluster of Excellence in Plant Sciences, Heinrich-Heine-University Düsseldorf, Düsseldorf, Germany
}

Keywords: floral transition, crop plants, Arabidopsis, phenological development, yield, evolution

\section{Editorial on the Research Topic}

\section{Recent Advances in Flowering Time Control}

The phenological development of plants can be broadly divided into 4 stages, embryo/juvenile, adult (all vegetative stages), reproductive (the generative stage), and senescent. This research topic focusses on the transition from vegetative growth to reproductive development, commonly referred to as floral transition. Plants have coordinated the seasonal timing of flowering and reproduction with the prevailing environmental conditions. In agriculture, flowering is a prerequisite for crop production whenever seeds or fruits are harvested. In contrast, avoidance of flowering is necessary for harvesting vegetative parts of a plant such as tubers or roots. Late flowering also severely hampers breeding success due to long generation times. Thus, flowering time regulation is of utmost importance for genetic improvement of crops.

In the past decades, we have gained increasing knowledge of flowering time regulation in model species such as Arabidopsis thaliana (Blümel et al., 2015). Genes coordinately regulating floral transition have been grouped into different pathways that have recently been illustrated in a WIKIPATHWAYS web interface (http://wikipathways.org//index.php?query=flowering\&title= Special\%3ASearchPathways\&doSearch=1\&sa=Search). Several of these pathways are activated by different environmental factors such as low temperature, day length, light intensity, or stress. Conservation of major flowering-time regulators and regulatory pathways between different species as well as increased availability of genome sequences and improvements in computational biology offer a unique opportunity to study flowering time genes across species. In general terms, the central elements that perceive day-length signals to control floral transition are conserved across the plant kingdom. CONSTANS (CO)-like genes and PHOSPHATIDYLETHANOLAMINE-BINDING PROTEIN (PEBP) encoding genes play major roles in these pathways and were first identified by genetic analysis in Arabidopsis. CO-like sequences seem to exist in all plants. Recent evidence indicates that $C O$ of Arabidopsis arose from a family-specific duplication and similar events might have occurred independently in many plant families (Simon et al., 2015). In contrast, plants from the Amaranthaceae family are lacking a true CO ortholog (Dally et al., 2014).

This research topic is focused on flowering time control in cultivated species. It contains nine review, perspective, and opinion articles and 14 original research articles which cover a large range of organisms from model species to crops.

New components have been added to the network of flowering time regulators mostly working upstream of key regulator elements, e.g., GATA transcription factors, small RNAs, in particular microRNAs (miRNAs) or sugar molecules. The GATA transcription factors GATA, NITRATE-INDUCIBLE, CARBON-METABOLISM INVOLVED (GCN), and GCN-like (GNL) previously identified as growth regulators mediating control by several phytohormones have 
emerged as repressors of flowering, acting via SOC1 (Richter et al., 2013; Behringer and Schwechheimer). During the juvenile to adult phase transition, a gradual decrease in miR156 and a reciprocal increase in miR172 ultimately leads to the activation of FLOWERING LOCUS T (FT) (Wang et al., 2009a; Wu et al., 2009). To facilitate the genome-wide analysis of small RNA-seq data, the DARIO tool developed for animals has been adapted for use in plants (plantDARIO) (Patra et al.).

The research topic also reflects the immense technical progress from the past years. Initially, flowering time regulators from crops were mainly cloned due to their sequence homology with known flowering time genes, mainly from Arabidopsis although the INDETERMINATE flowering gene was cloned from maize by transposon tagging (Colasanti et al., 1998). Later, new genes were identified from crops using flowering time QTLs (quantitative trait loci) by map-based cloning approaches. Now, whole genome or candidate gene association mapping and transcriptome analysis have become important strategies (Schiessl et al.). For instance, whole transcriptome analysis revealed the circadian clock homolog of EARLY FLOWERING 3 (ELF3) and mapping-by-sequencing applied on exome-capture data from phenotypic bulks identified PHYTOCHROME $C$ as important components of photoperiodic flowering in barley (Faure et al., 2012; Pankin et al., 2014). In addition, the wild barley nested association mapping population HEB-25 was used to associate major flowering time genes with phenological development in different field environments (Maurer et al., 2015, 2016). Furthermore, several flowering time genes like $P p d-$ H1 (PRR37) and HvSDW1 (GA20ox2) were linked to both, developmental and yield-related traits. In a salinity tolerance study with HEB-25, the wild barley allele at the HvCEN locus (Antirrhinum CENTRORADIALIS, TFL1-like) promoted flowering and maturity, resulting in a higher harvest index and a higher yield under salt stress in the field (Saade et al., 2016). These findings indicate that searching for allelic variants of known flowering time genes, also taken from related wild species, may substantially support future plant breeding efforts to increase plant performance under optimal cultivation conditions as well as under stress.

Flowering time regulation is strongly conserved among the Brassicas to which Arabidopsis also belongs. Two articles (Guo et al.; Schiessl et al.) describe flowering time genes from oilseed rape where the vernalization pathway with its central element FLOWERING LOCUS C (FLC) is essentially the same as in Arabidopsis. In contrast, an $F L C$ homolog from beet was proven not to be a major regulator of vernalization response in biennial beets Vogt et al. New sequence variation has been induced in rapeseed by EMS mutagenesis which gave rise to plants with altered flowering time in spite of the polyploid nature of this species. Mutations within a single gene can have a big impact on flowering time even if there are several paralogs of an Arabidopsis flowering time gene present in the rapeseed genome.

The research topic demonstrates that the range of model species has been constantly increased to allow a broader range of flowering-related traits to be studied. Arabis alpina and Brachypodium distachyon serve as models for perennials (Wang et al., 2009b) and for grasses Woods et al., respectively. A recent overview on flowering regulation in grass species is given in this research topic (Fjellheim et al.). The authors discuss molecular pathways that control seasonal flowering responses in the Pooideae sub-family and how variations in flowering time gene activities contributed to the adaptation to different environments. Refined flowering time regulatory pathways have been identified from rice (Shrestha et al., 2014) and barley/wheat (Chen et al., 2014) (Mulki and von Korff, 2016). Loscos et al. show that natural allelic variation in copy number of the florigen HvFT1 is present in European spring barley cultivars lacking a vernalization requirement to initiate flowering (Loscos et al., 2014). However, no clear relationship between HvFT1 copy number and expression was observed in a set of diverse spring barley genotypes.

Some articles from this research topic highlight multiple functions of flowering time genes beyond floral transition. These genes impact multiple developmental processes and they are regulators of yield components and stress tolerance (Kazan and Lyons, 2016). In this respect, members of the PHOSPHATIDYLETHANOLAMINE-BINDING PROTEIN (PEBP) gene family, such as FLOWERING LOCUS T (FT) and TERMINAL FLOWER 1 (TFL) of Arabidopsis attracted the highest attention. As highlighted for tomato (Lifschitz et al.) and for rice (Izawa et al., 2016) fine tuning of the $S F T / S P$ (which are true orthologs of FT and TFL) ratio is an important process for patterning plant architecture. Lifschitz et al. explain how the ratios between FT-like and TFL1like genes control the patterning of the shoot systems across many different plants. In tomato, an increase of the florigen protein SINGLE FLOWER TRUSS (SFT, FT-like), relative to the anti-florigen protein SELF PRUNING (SP, TFL1-like) induces growth arrest and termination of meristems across the tomato shoot, while high relative levels of $S P$ promote the formation of an indeterminate vegetative inflorescence. Consequently, $S F T / S P$ ratios determine the number of flowers and eventually, tomato fruits per inflorescence. Naturally occurring mutations have been selected in both gene families to adapt crops to different environments and to increase productivity. TFL1 mutants have been frequently used in breeding (e.g., tomato, soybean, roses, and barley). Likewise, mutations of FT-like genes were selected in sunflower, sugar beet, rice, potato, and wheat. Moreover, single point mutations within FT- and TFL1 orthologs can drastically alter their function from floral inducers to floral repressors, as demonstrated for sugarcane Coelho et al. and beet (Pin et al., 2010). We propose that selecting for FT/TFL1 sequence variations in crop plants may pave the way to further improvements in elite crop productivity.

\section{AUTHOR CONTRIBUTIONS}

All authors listed, have made substantial, direct and intellectual contribution to the work, and approved it for publication.

\section{FUNDING}

We gratefully acknowledge funding by the German Research Foundation (Priority Program 1530 and Deutsche Forschungsgemeinschaft). 


\section{REFERENCES}

Blümel, M., Dally, N., and Jung, C. (2015). Flowering time regulation in crops - what did we learn from Arabidopsis? Curr. Opin. Biotechnol. 32, 121-129. doi: 10.1016/j.copbio.2014.11.023

Chen, A., Li, C., Hu, W., Lau, M. Y., Lin, H., Rockwell, N. C., et al. (2014). PHYTOCHROME C plays a major role in the acceleration of wheat flowering under long-day photoperiod. Proc. Natl. Acad. Sci. U.S.A. 111, 10037-10044. doi: 10.1073/pnas.1409795111

Colasanti, J., Yuan, Z., and Sundaresan, V. (1998). The indeterminate gene encodes a zinc finger protein and regulates a leaf-generated signal required for the transition to flowering in maize. Cell 93, 593-603. doi: 10.1016/s0092-8674(00)81188-5

Dally, N., Xiao, K., Holtgräwe, D., and Jung, C. (2014). The B2 flowering time locus of beet encodes a zinc finger transcription factor. Proc. Natl. Acad. Sci. U.S.A. 111, 10365-10370. doi: 10.1073/pnas.1404829111

Faure, S., Turner, A. S., Gruszka, D., Christodoulou, V., Davis, S. J., von Korff, M., et al. (2012). Mutation at the circadian clock gene EARLY MATURITY 8 adapts domesticated barley (Hordeum vulgare) to short growing seasons. Proc. Natl. Acad. Sci. U.S.A. 109, 8328-8333. doi: 10.1073/pnas.1120496109

Izawa, T., Okada, R., Endo, N., Nemoto, Y., Takamizo, T., Tsuzuki, S., et al. (2016). Poaceae Plant whose Flowering Time is Controllable. Tsukuba: United States Patent.

Kazan, K., and Lyons, R. (2016). The link between flowering time and stress tolerance. J. Exp. Bot. 67, 47-60. doi: 10.1093/jxb/erv441

Loscos, J., Igartua, E., Contreras-Moreira, B., Gracia, M. P., and Casas, A. M. (2014). HvFT1 polymorphism and effect-survey of barley germplasm and expression analysis. Front. Plant Sci. 5:251. doi: 10.3389/fpls.2014.00251

Maurer, A., Draba, V., Jiang, Y., Schnaithmann, F., Sharma, R., Schumann, E., et al. (2015). Modelling the genetic architecture of flowering time control in barley through nested association mapping. BMC Genomics 16:290. doi: 10.1186/s12864-015-1459-7

Maurer, A., Draba, V., and Pillen, K. (2016). Genomic dissection of plant development and its impact on thousand grain weight in barley through nested association mapping. J. Exp. Bot. 67, 2507-2518. doi: 10.1093/jxb/erw070

Mulki, M. A., and von Korff, M. (2016). CONSTANS controls floral repression by up-regulating VERNALIZATION2 (VRN-H2) in barley. Plant Physiol. 170, 325-337. doi: 10.1104/pp.15.01350

Pankin, A., Campoli, C., Dong, X., Kilian, B., Sharma, R., Himmelbach, A., et al. (2014). Mapping-by-sequencing identifies HvPHYTOCHROME $\mathrm{C}$ as a candidate gene for the early maturity 5 locus modulating the circadian clock and photoperiodic flowering in barley. Genetics 198, 383-396. doi: 10.1534/genetics.114.165613
Pin, P. A., Benlloch, R., Bonnet, D., Wremerth-Weich, E. S. I., Kraft, T., Gielen, J. J., et al. (2010). An antagonistic pair of $F T$ homologs mediates the control of flowering time in sugar beet. Science 330, 1397-1400. doi: 10.1126/science.1197004

Richter, R., Behringer, C., Zourelidou, M., and Schwechheimer, C. (2013). Convergence of auxin and gibberellin signaling on the regulation of the GATA transcription factors GNC and GNL in Arabidopsis thaliana. Proc. Natl. Acad. Sci. U.S.A. 110, 13192-13197. doi: 10.1073/pnas.13042 50110

Saade, S., Maurer, A., Shahid, M., Oakey, H., Schmöckel, S. M., Negrão, S., et al. (2016). Yield-related salinity tolerance traits identified in a nested association mapping (NAM) population of wild barley. Sci. Rep. 6: 32586. doi: 10.1038/srep 32586

Shrestha, R., Gómez-Ariza, J., Brambilla, V., and Fornara, F. (2014). Molecular control of seasonal flowering in rice, arabidopsis and temperate cereals. Ann. Bot. 114, 1445-1458. doi: 10.1093/aob/mcu032

Simon, S., Rühl, M., De Montaigu, A., Wötzel, S., and Coupland, G. (2015). Evolution of CONSTANS regulation and function after gene duplication produced a photoperiodic flowering switch in the brassicaceae. Mol. Biol. Evol. 32, 2284-2301. doi: 10.1093/molbev/msv110

Wang, J. W., Czech, B., and Weigel, D. (2009a). miR156-regulated SPL transcription factors define an endogenous flowering pathway in Arabidopsis thaliana. Cell 138, 738-749. doi: 10.1016/j.cell.2009. 06.014

Wang, R. H., Farrona, S., Vincent, C., Joecker, A., Schoof, H., Turck, F., et al. (2009b). PEP1 regulates perennial flowering in Arabis alpina. Nature 459, U423-U138. doi: 10.1038/nature07988

Wu, G., Park, M. Y., Conway, S. R., Wang, J. W., Weigel, D., and Poethig, R. S. (2009). The sequential action of miR156 and miR172 regulates developmental timing in Arabidopsis. Cell 138, 750-759. doi: 10.1016/j.cell.2009. 06.031

Conflict of Interest Statement: The authors declare that the research was conducted in the absence of any commercial or financial relationships that could be construed as a potential conflict of interest.

Copyright (๑ 2017 Jung, Pillen, Staiger, Coupland and von Korff. This is an openaccess article distributed under the terms of the Creative Commons Attribution License (CC BY). The use, distribution or reproduction in other forums is permitted, provided the original author(s) or licensor are credited and that the original publication in this journal is cited, in accordance with accepted academic practice. No use, distribution or reproduction is permitted which does not comply with these terms. 